\title{
MEMS Non-Absorbing Electromagnetic Power Sensor Employing the Effect of Radiation Pressure ${ }^{\dagger}$
}

\author{
Ivan Ryger 1,2,*, Alexandra Artusio-Glimpse ${ }^{3}$, Paul Williams ${ }^{3}$, Gordon Shaw ${ }^{4}$, \\ Matthew Simons ${ }^{3}$, Christopher Holloway ${ }^{3}$ and John Lehman ${ }^{3}$ \\ 1 Associate of the National Institute of Standards and Technology, Boulder, CO 80305, USA \\ 2 Department of Physics, University of Colorado, Boulder, CO 80309, USA \\ 3 National Institute of Standards and Technology, 325 Broadway, Boulder, CO 80305, USA; \\ alexandra.artusio-glimpse@nist.gov (A.A.-G.); paul.williams@nist.gov (P.W.); \\ matthew.simons@nist.gov (M.S.); christopher.holloway@nist.gov (C.H.); john.lehman@nist.gov (J.L.) \\ 4 National Institute of Standards and Technology, 100 Bureau Dr, Gaithersburg, MD 20899, USA; \\ gordon.shaw@nist.gov \\ * Correspondence: ivan.ryger@nist.gov; Tel.: +1-303-497-5795 \\ + Presented at the Eurosensors 2018 Conference, Graz, Austria, 9-12 September 2018.
}

Published: 24 December 2018

\begin{abstract}
We demonstrate a compact electromagnetic power sensor based on force effects of electromagnetic radiation onto a highly reflective mirror surface. Unlike the conventional power measurement approach, the photons are not absorbed and can be further used in the investigated system. In addition, the exerted force is frequency-independent, yielding a wide measurement frequency span being practically limited by the wavelength-dependent mirror reflection coefficient. The mechanical arrangement of two sensing elements in tandem suppresses the influence of gravity and vibrations on the power reading. We achieve a noise floor of about $1 \mathrm{~W} / \mathrm{VHz}$ and speed of $100 \mathrm{~ms}$, being practically limited by sensor's dynamics and lock-in amplifier filter settling time.
\end{abstract}

Keywords: radiation pressure; capacitive sensor; lock-in amplifier; silicon micromachining; acoustic noise suppression; tilt immunity; distributed bragg reflector; Archimedian spiral spring

\section{Introduction}

The industrial use of electromagnetic power sources (e.g., laser-based additive manufacturing, and microwave plasma processes in nanotechnology) is constantly rising. Accurate knowledge of delivered power is vital for understanding the underlying physical processes and simultaneously ensuring consistent process quality. The most accurate primary standards for absolute SI-traceable measurement of high electromagnetic (EM) power at National Institute of Standards and Technology are based on comparison of heat generated by absorption of the EM energy to its SI-traceable Joule electrical equivalent [1]. Calorimeters used for this purpose require a total absorption of electromagnetic energy in a black body rendering it unavailable for further use. They are from their physical nature bulky and slow instruments integrating out short-term fluctuations of the measured power. On the other hand, often industrial EM power sources are measured by a small portion of the energy being picked off by a power splitter. However, due to fluctuations of the splitting ratio, the measurement error is oftentimes at the level of measurand [2]. For this reason, there exists an open gap between high precision primary power standards for EM power and compact and fast industrial sensors (often photodetectors in laser applications). Measuring power by means of radiation pressure is attractive because it stems from the linear and frequency-independent relationship between the incident laser power and the force it exerts on a mirror (1) 


$$
F=2 P r \cos (\theta) / c
$$

where $F, P, \theta$ and $c$ are force applied onto mirror, optical power, angle of incidence towards plane normal and speed of the light, respectively. The reflectivity dependent constant $r=R+(1-R) \alpha / 2$ accounts for light momentum transfer in the case of finite reflectivity $R$ and absorptivity $\alpha$ of the mirror [3]. The measurement of the force effects of EM radiation has been carried out successfully in many publications [4-8]. However, these laboratory instruments were found to be delicate and sensitive to environmental conditions and thus impractical for routine operation. But now, owing to the availability of high-sensitivity industrial scales and dielectric mirrors that can be optimized for very high reflectivity (typically $>99.99 \%$ for optical and $>90 \%$ for RF), a SI-traceable high optical power measurement by means of the force exerted by photons onto a highly reflective mirror has been demonstrated [3]. This principle allows an energy-carrying beam to be bounced off a measurement mirror and still used in the target application. However, this concept suffers from an inherent sensitivity to inertial forces (acoustic vibrations and tilt-dependent projection of gravity). As a proposed solution, we demonstrate a miniaturized version of a radiation pressure sensor that could potentially bridge the gap between the accurate primary EM power standards and better-performing but less accurate industrial detectors. We describe here a design to inherently suppress the environmental effects on the sensor reading, thus approaching the needs of manufacturing floor. Its compact size and ability of direct force calibration are enabling features for its use inside sources of electromagnetic energy, making a considerable paradigm shift from calibrated detectors towards calibrated energy sources. We demonstrate the performance of this sensor to measure microwave power at a frequency of $15 \mathrm{GHz}$.

\section{Sensor Design and Characterization}

The sensor consists of a silicon micromachined parallel plate capacitor, both of whose disk electrodes (diameter $20 \mathrm{~mm}$ ) are attached to a rigid frame through weak springs (width $465 \mu \mathrm{m}$, thickness $380 \mu \mathrm{m}$, length $45 \mathrm{~mm}$ ) in the shape of an Archimedean spiral (Figure 1a). For the optical sensor, a highly reflective dielectric mirror (optimized for reflectance with a value of $0.9992 \pm 0.0002$ at $1.07 \mu \mathrm{m}$ wavelength and $45^{\circ}$ angle of incidence) is deposited onto the front side of one disk electrode. The rear side of the silicon chip is covered by continuous $\mathrm{Ti} / \mathrm{Au}$ metallization to prevent photoconductive effects from affecting the capacitance reading. An additional optimized Ti/Au layer is deposited on top of the Archimedian springs to compensate the bimorph thermal bending of springs. Two identical chips are separated by thin $(\sim 2.5 \mu \mathrm{m})$ insulating polyimide foil with metallized electrical contacts (Figure $1 \mathrm{~b}$ shows such a device for laser power measurement) and clamped together. In the case of a microwave power sensor, Figure 1c shows a setup with a front mirror made from an aluminum foil approximately $30 \mu \mathrm{m}$ thick. Due to our lack of available sensor chips at the time, for this microwave sensor, the bottom element of the sensor was replaced by a machined aluminum plate (Figure 1c).

To obtain a calibration between a measured electrical signal and the applied optical power, an accurate knowledge of electro-mechanical parameters (spring constant, capacitor plate spacing and capacitance gradient with respect to plate spacing) of our transducer is needed. For this purpose, we developed an opto-electronic characterization technique, where we apply a time-varying electrostatic bias voltage to the capacitor's electrodes and measure the axial displacement of the mirrors [9]. Simultaneously we record the geometrical capacitance measured by a LCR meter as a function of capacitor plate spacing (Figure 2a), yielding the knowledge of the capacitance gradient. We employ a dual axis heterodyne interferometer to measure the displacements of both front and back mirror faces. The optical setup comprises two single pass interferometers in a cat's-eye arrangement [10], that have been proven to be relatively insensitive to tilt errors. Using these measurements, we determine the initial spacing of the capacitor's electrodes, the mechanically inactive (parasitic) capacitance and the spring constant.

To demonstrate the benefits of dual spring tandem arrangement we mounted the sensors from Figure $1 b, c$ onto rotational stage and recorded the sensor's geometrical capacitance as a function of 
tilt angle. Then this value was mapped into equivalent plate spacing based on known capacitance calibration (Figure 2a). We measure suppression of tilt-dependent gravity projection by factor of 100 in the case of two identical silicon chips operating in tandem compared to a device with second electrode being a rigid aluminum plate.

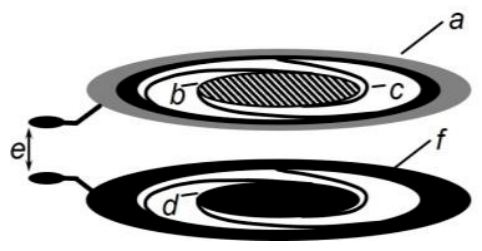

(a)

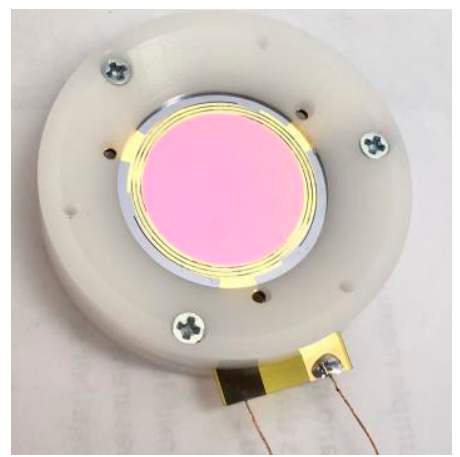

(b)

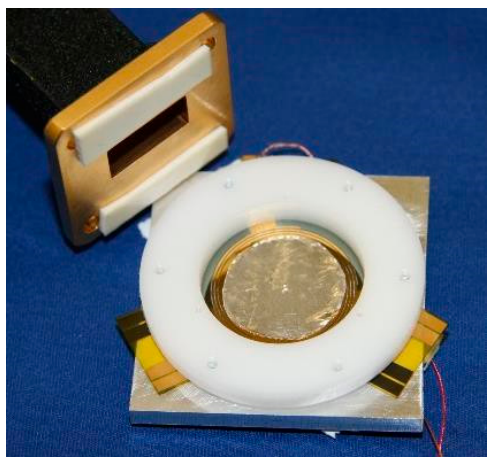

(c)

Figure 1. (a) Schematic view of the dual spring radiation pressure sensor. It consists of two disk electrodes, $a$ with mirror on top $b$ and a metallic coating $d$ on the back side. These moving disks are attached to the supporting annulus $a$ through three Archimedian springs $c$. Electrical measurement is provided through contacts $e$. (b) Photograph of fabricated dual spring optical sensor. (c) Microwave power sensor with bottom element replaced by an aluminum plate, next to the WR62 waveguide.

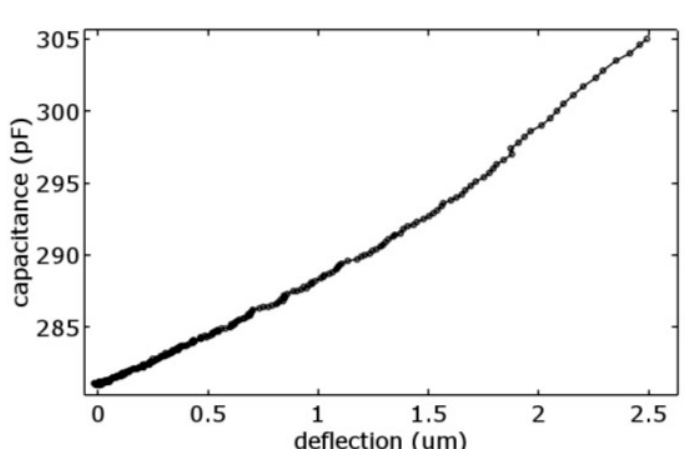

(a)

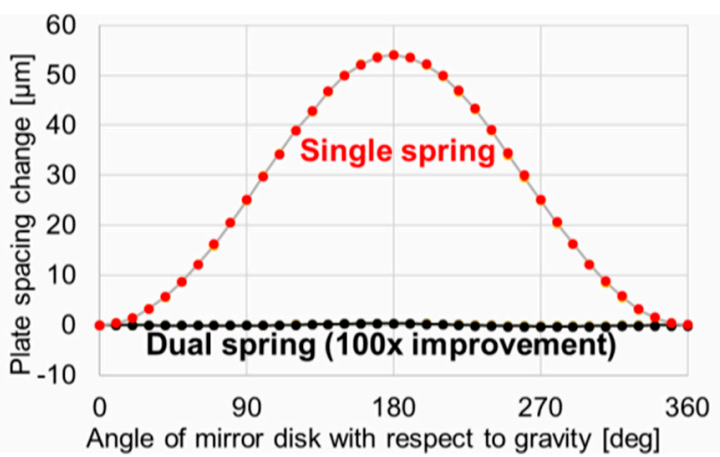

(b)

Figure 2. (a) Plot of the sensor's geometrical capacitance as a function of deflection. This is to determine the capacitance gradient, parasitic capacitance and initial plate spacing (comparison of two methods). (b) Plot of capacitor plate spacing as a function of the tilt angle. Zero angle corresponds to moveable mirror surface facing upwards and fixed plate downwards, $180^{\circ}$ angle corresponds to fixed plate facing upwards and moveable electrode facing downwards.

\section{Microwave Power Experiment}

Due to the lack of available chips during the test period we used a single spring device with machined aluminum backplate instead of dual spring device. The sensor was mounted directly onto the output flange of WR62 waveguide. The output bridge signal (proportional to plate capacitance) was fed to a dual phase lock-in amplifier and its output was recorded by a digital storage oscilloscope. The microwave power at $15 \mathrm{GHz}$ was provided by a signal generator connected to a travelling wave tube amplifier. Two waveguide ferrite isolators were placed in the signal path to dissipate the power reflected from the sensor. The output power was monitored with a directional coupler which picked off a fraction of the microwave power reflecting from the mirror, further attenuated it and measured it with a bolometer power head. The signal generator was modulated by a square wave of $1 \mathrm{~Hz}$ frequency and $50 \%$ duty cycle. To decrease the contribution of the un-correlated noise we triggered the oscilloscope from the same modulation source and used waveform averaging [9]. The experiment was performed in a Faraday cage padded with foam microwave absorbers. To monitor the drift of the sensor, we measured its temperature during the experiment by an attached thermocouple 
thermometer with a readout outside the measurement chamber. A plot of the transient response of the sensor is shown in Figure 3a. The linearity of the measurement is evaluated by plotting the sensor response as a function of power in Figure $3 b$.

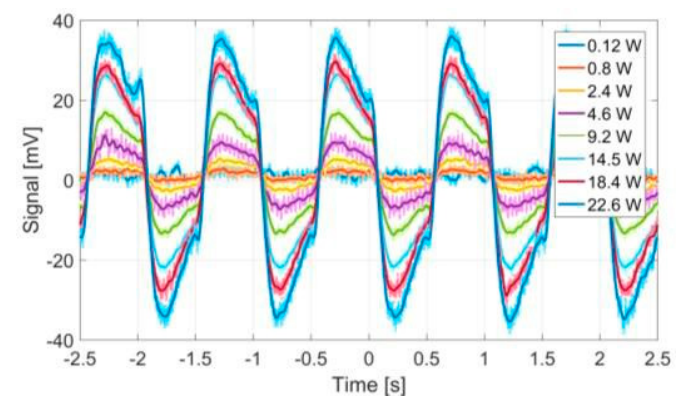

(a)

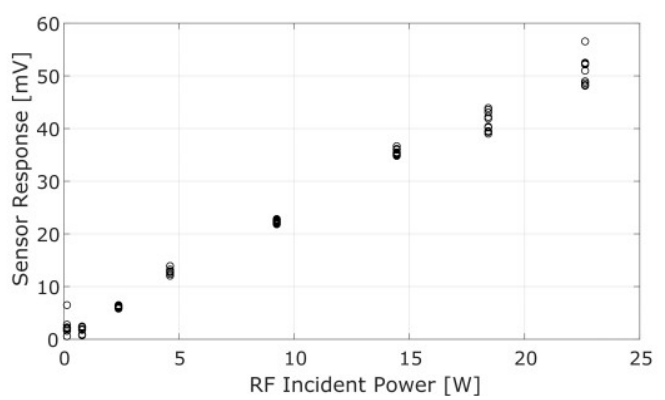

(b)

Figure 3. (a) Transient response of the miniature radiation pressure power meter to a microwave power. The slow creep of the sensor baseline is attributed to a high pass filter at the input of the oscilloscope (AC coupling). (b) Plot of the sensor's response peak signal against varying incident power; following the linear trend.

\section{Conclusions}

In this contribution we demonstrated the manufacturing process and characterization of a MEMS power sensor for detecting the force effects of EM radiation. We demonstrated its performance by measuring the output power of microwave frequency radiation. We show an achieved noise limit of about $1 \mathrm{~W} / \sqrt{\mathrm{Hz}}$ and response time around $100 \mathrm{~ms}$ that is limited by sensor mechanics and the lockin amplifier's filter settings. We also demonstrate benefits of tandem spring arrangement, suppressing the tilt-dependent projection of gravity force by factor of 100 . Owing to its small mechanical dimensions, noise-rejecting dual spring topology and optimized design, this device extends the applicable measurement range towards lower power levels and broad frequency range.

Disclaimer: This publication is a contribution of the U.S. government and is not subject to copyright. Identification of commercial items is for clarity and does not represent an endorsement by NIST.

\section{References}

1. Williams, P.A.; Hadler, J.A.; Cromer, C.; West, J.; Li, X.; Lehman, J.H. Flowing-water optical power meter for primary-standard, multi-kilowatt laser power measurements. Metrologia 2018, 55, 427-436.

2. Spidell, M.; Hadler, J.A.; Stephens, M.; Williams, P.; Lehman, J.H. Geometric contributions to chopper wheel optical attenuation and uncertainty. Metrologia 2017, 54, L19-L25.

3. Williams, P.; Hadler, J.A.; Maring, F.C.; Lee, R.; Rogers, K.A.; Simonds, B.J.; Spidell, M.T.; Feldman, A.D.; Lehman, J.H. Portable, high-accuracy, non-absorbing laser power measurement at kilowatt levels by means of radiation pressure. Opt. Express 2017, 25, 4382-4392.

4. Nichols, E.F.; Hull, G.F. A7 preliminary communication on the pressure of heat and light radiation. Phys. Rev. Ser. I 1901, 13, 307-320.

5. Lebedev, P.N. Experimental examination of light pressure. Ann. Phys. 1901, 6, 1-26.

6. Cullen, A.L. Absolute power measurement at microwave frequencies. Proc. IEEE 1952, 92, 100-111.

7. Ma, D.; Garrett, J.L.; Munday, J.N. Quantitative measurement of radiation pressure on microcantilever in ambient environmnent. Appl. Phys. Lett. 2015, 106, 091107.

8. Agatsuma, K.; Friedrich, D.; Ballmer, S.; DeSalvo, G.; Sakata, S. Nishida, E. Kawamura, S. Precise measurement of laser power using an optomechanical system. Opt. Express 2014, 22, 2013-2030. 
9. Ryger, I.; Artusio-Glimpse, A.B.; Williams, P.; Tomlin, N.; Stephens, M.; Rogers, K.; Spidell, M.; Lehman, J., Micromachined force scale for optical power measurement by radiation pressure sensing. IEEE Sens. J. 2018, 18, 7941-7948.

10. Peña-Arellano, F.E.; Speake, C.C. Mirror tilt immunity interferometry with a cat's eye retroreflector. Appl. Opt. 2011, 50, 981-991. article distributed under the terms and conditions of the Creative Commons Attribution (CC BY) license (http://creativecommons.org/licenses/by/4.0/). 